\title{
Remoção do 2,4-D em amostras de águas pela adsorção em leitos fixos de carvão ativado granular em escala reduzida
}

\author{
Removal of 2,4-D in water samples by adsorption in \\ fixed beds of granular activated carbon on reduced scale
}

Edumar Ramos Cabral Coelho"* (1), Andréia do Rozário²

\begin{abstract}
RESUMO
O objetivo deste trabalho foi avaliar, por meio dos testes rápidos de coluna em escala reduzida, a capacidade de adsorção do carvão ativado granular (CAG) na remoção do ácido 2,4-diclorofenoxiacético (2,4-D) em amostras de água ultrapura e de água filtrada proveniente de estação de tratamento de água. Os valores da capacidade de adsorção obtidos a partir de amostras de água ultrapura foram $67 \%$ superiores aos obtidos para água com presença de matéria orgânica natural (MON). A MON influenciou fortemente a remoção de 2,4-D competindo pelos sítios de adsorção do carvão ativado e diminuiu a capacidade de adsorção para 7,43 mg 2,4-D por grama de CAG. Para a concentração de 2,4-D no afluente igual a $7089 \pm 104 \mu \mathrm{g} \mathrm{L}^{-1} \mathrm{e}$ e relação volume ${ }_{\text {água }} /$ volume $_{\text {CAG }}$ estimado em 766, foi possível produzir água com concentração de 2,4-D inferior a $30 \mu \mathrm{g} \cdot \mathrm{L}^{-1}$, como recomendado pela Portaria Brasileira de Potabilidade de Água, do Ministério da Saúde (Portaria MS no 2.914/2011).
\end{abstract}

Palavras-chave: adsorção; 2,4-D; carvão ativado granular; matéria orgânica natural; testes rápidos de coluna em escala reduzida.

\begin{abstract}
The aim of this study was to assess by means of rapid tests of smallscale column the adsorption capacity of granular activated carbon (GAC) for removal of 2,4-dichlorophenoxyacetic acid (2,4-D) from samples of ultrapure water and filtered water from a water treatment plant. The adsorption capacities values obtained from distilled water samples were $67 \%$ higher than those obtained for water having natural organic matter (NOM). NOM strongly influenced the removal of 2,4-D, competing for GAC adsorption sites, and decreasing its adsorption capacity to $7 \mathrm{mg}$ 2,4-D g $\mathrm{g}^{-1}$ CAG. For 2,4-D concentration of $7089 \pm 104 \mu \mathrm{g} \mathrm{L}^{-1}$ and the water treated volume/GAC volume at 766 , it was possible to produce treated water with 2,4-D concentration less than $30 \mu \mathrm{g} \mathrm{L}^{-1}$ as recommended by Brazilian Drinking Water Legislation of the Ministry of Health (Portaria MS 2914/2011).
\end{abstract}

Keywords: adsorption; 2,4-D; granular activated carbon; natural organic matter; Rapid Small-Scale Column Tests.

\section{INTRODUÇÃO}

O papel cada vez mais relevante dos agrotóxicos na produção de alimentos em larga escala tem resultado na ampliação de seu uso em nível mundial. O impacto ambiental dos agrotóxicos no meio ambiente e na saúde pública tem sido reportado por vários pesquisadores. Na saúde, podem causar danos ao sistema reprodutor e têm também papel estabelecido como precursores de câncer. Segundo as informações fornecidas pela base de dados da Pesticide Action Network (PAN, 2010), o ácido 2,4-diclorofenoxiacético (2,4-D) apresenta toxicidade moderada, é potencialmente cancerígeno e suspeito de causar alterações endócrinas.
A Portaria do Ministério da Saúde (MS) nº 2.914/2011 (BRASIL, 2011) estabelece, para somatória dos ácidos 2,4-D e 2,4,5-triclorofenoxiacético (2,4,5-T), o valor máximo permitido de $30 \mu \mathrm{g} . \mathrm{L}^{-1}$. No Brasil, a Agência Nacional de Vigilância Sanitária (ANVISA) classificou o 2,4-D como extremamente tóxico (Classe I) para a saúde (BRASIL, 2003a).

O Brasil destaca-se no cenário mundial como o maior consumidor de agrotóxicos da América Latina, respondendo por $86 \%$ do consumo desses produtos (IBGE, 2015). Nesse contexto, a classe de agrotóxicos mais intensamente aplicada é a dos herbicidas (mais de 50\% do total), seguida pelos inseticidas, fungicidas e acaricidas. Entre os herbicidas,

'Universidade Federal do Espírito Santo - Vitória (ES), Brasil.

IInstituto Federal de Educação, Ciência e Tecnologia do Espírito Santo - Vitória (ES), Brasil.

*Autor correspondente: edumar.coelho@ufes.br

Recebido: 19/07/2017 - Aceito: 13/04/2018 - Reg. ABES: 182897 
um dos princípios ativos mais utilizados é o 2,4-D, autorizado no Brasil para aplicação nas culturas de soja (em pré-plantio), milho, cana-de-açúcar, café, trigo, aveia, centeio, arroz e pastagem formada (IBAMA, 2010). Possui caráter polar e ácido, pKa igual a 2,87; solubilidade em água igual a $900 \mathrm{mg} \cdot \mathrm{L}^{-1}$ a $25^{\circ} \mathrm{C}$, massa molar de 21,02 g.mol ${ }^{-1}$ e largura molecular de 2,074 Å (CHINGOMBE; SAHA; WAKEMAN, 2006). Sua fórmula estrutural está representada na Figura 1.

Segundo Stackelberg et al. (2007) e Jin e Peldszuz (2012), o processo de tratamento de água por ciclo completo não tem sido efetivo na remoção de microcontaminantes, tais como o 2,4-D. Entre as tecnologias propostas para remoção de microcontaminantes, a adsorção em carvão ativado é considerada uma das mais efetivas para remoção de substâncias causadoras de gosto e odor, compostos orgânicos sintéticos, substâncias formadoras de cor, subprodutos de desinfecção, constituintes inorgânicos e agrotóxicos (CORWIN; SUMMERS, 2012; REN et al., 2011; SALMAN; NJOKU; HAMEED, 2011).

O carvão ativado é constituído de uma estrutura composta de macroporos, mesoporos e microporos e possui grande área superficial, o que o torna atrativo para remoção de moléculas orgânicas presentes em águas para abastecimento. As propriedades físicas de superfície são frequentemente influenciadas pela área superficial específica (ASE) e pelo tamanho dos poros, enquanto as propriedades químicas dependem da natureza de grupos ácidos ou básicos e, também, da presença ou ausência desses grupos sobre sua superfície. Para o adsorvato, as principais características que influenciam na sua adsorção são: o tamanho molecular, a polaridade e as características ácidas ou básicas, medidas pelas constantes de acidez $(\mathrm{Ka})$ ou de basicidade $(\mathrm{Kb})$. No caso de um adsorvato como o 2,4-D, a natureza dos grupos substituintes dos anéis aromáticos também é um fator de influência (CASTILLA, 2004).

A remoção de contaminantes químicos, pelo processo de adsorção, é influenciada pelas propriedades do adsorvente e do adsorvato, pela temperatura, pelo tempo de contato e pelas características do meio líquido com suas espécies competitivas (JIANG; ADAMS, 2006). Em águas naturais, o processo de adsorção apresenta limitação pela presença de substâncias orgânicas e inorgânicas, que poderão competir com o adsorvato

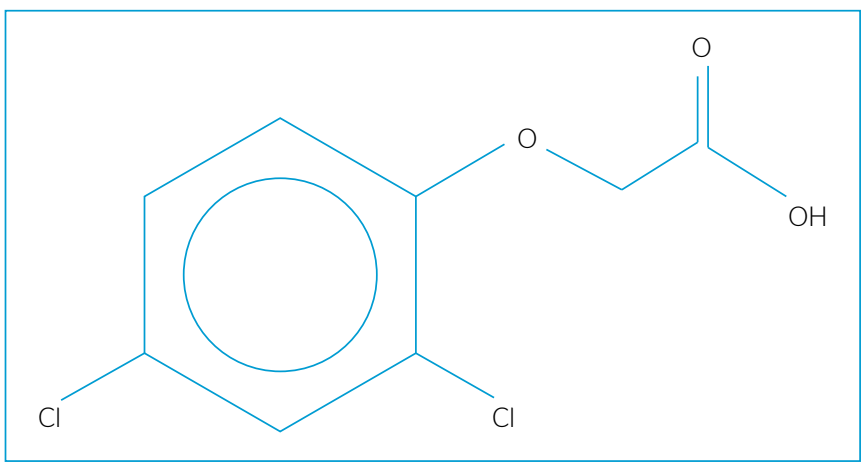

Figura 1 - Fórmula estrutural plana do ácido 2,4-diclorofenoxiacético. pelos sítios ativos do carvão ou bloquear os seus poros (ERSAN et al., 2016). A matéria orgânica natural (MON) de baixo peso molecular tem sido apontada como a principal causa de competição, enquanto a de maior peso molecular causa bloqueio dos poros, impedindo acesso do microcontaminante aos microporos (HUMBERT et al., 2008). Por causa das variações das características da $\mathrm{MON}$ em águas naturais, torna-se difícil prever a extensão da adsorção e a competição em sistemas multicomponentes, sendo necessária a obtenção experimental de isotermas de adsorção ou a execução de testes de adsorção em colunas.

A adsorção em leitos fixos de carvão ativado granular (CAG) permite o contato contínuo entre o adsorvato e adsorvente, resultando em aumento da utilização do adsorvente, maior facilidade no controle do processo e melhor relação custo versus eficiência, quando comparado à aplicação do carvão ativado pulverizado (SCHIDEMAN et al., 2007; SCHARF et al., 2010). De acordo com Heijman e Hopman (2009), se a relação entre o volume de água tratada e o volume de carvão ativado é a mesma e a competição entre o agrotóxico é também considerada constante, as condições cinéticas e de competição estão combinadas em um único experimento, sendo suficiente a predição da curva de ruptura.

No dimensionamento e na determinação da capacidade de adsorção de sistemas de CAG, além da estação piloto, podem ser utilizados os testes rápidos de colunas em pequena escala ou escala reduzida (rapid small-scale column tests - RSSCT). Espera-se que os resultados dos testes em pequena escala produzam curva de ruptura semelhante à curva que ocorreria em escala real. Segundo Crittenden et al. (1987), a principal vantagem dos testes em pequena escala é que o tempo de ensaio é minimizado para atingir a curva de ruptura. Além disso, garante-se o uso de uma amostra de água coletada no mesmo dia, evitando variações significativas em suas propriedades físico-químicas (MATSUI; KNAPPE; TAKAGI, 2002). A norma da American Society for Testing and Materials (ASTM) D 6586-03 (ASTM, 2008) estabelece critérios para a realização dos ensaios em RSSCT para determinação da curva de ruptura.

O objetivo do presente estudo foi avaliar a remoção do 2,4-D em amostras de águas de diferentes qualidades por meio da adsorção em leitos fixos de CAG, com o uso dos RSSCT, para determinação do tempo de ruptura e da capacidade de adsorção na produção de efluente com valor igual ou inferior a $30 \mu \mathrm{g} . \mathrm{L}^{-1}$, estabelecido pela Portaria MS no 2.914/2011 (BRASIL, 2011).

\section{METODOLOGIA}

\section{Caracterização do carvão ativado granular e das amostras de água}

O CAG utilizado na pesquisa foi obtido comercialmente, sendo produzido, segundo dados do fabricante, a partir da casca de coco por 
meio do processo de ativação física. Esse CAG possuía granulometria na faixa de 60 a $80 \mathrm{Mesh}$ (entre 0,177 e 0,250 mm), conforme sugerido por Crittenden, Berrigan e Hand (1986).

O material foi caracterizado para os seguintes parâmetros:

- densidade aparente: ASTM D 2854 (ASTM, 1996);

- ASE: Brunauer, Emmett e Teller (BET) - $\mathrm{N}_{2}$ a 77K;

- distribuição de porosidade: quenched solid density functional theory (QSDFT);

- número de iodo: ASTM D 4607 (ASTM, 1994);

- spectroscopia no infravermelho (Fourier transform infrared spectroscopy - FTIR);

- pH da água de lavagem do CAG: ASTM D 6851 (ASTM, 2002).

A preparação do carvão para montagem das colunas seguiu as recomendações da ASTM D 6586 (ASTM, 2008).

Os experimentos foram conduzidos em dois tipos de água, com adição de 2,4-D, para verificar a influência da MON na capacidade de adsorção. No primeiro ensaio foi utilizada água ultrapura e no segundo, água filtrada proveniente de estação de tratamento de água (ETA) após tratamento por filtração direta descendente. Durante os ensaios, a temperatura do laboratório foi mantida em $21 \pm 1^{\circ} \mathrm{C}$.

Os parâmetros físico-químicos de caracterização foram $\mathrm{pH}$, turbidez, condutividade, absorbância a $254 \mathrm{~nm}$, cor verdadeira e alcalinidade, utilizando-se as metodologias estabelecidas pelo Standard Methods for the Examination of Water and Wastewater (APHA; AWWA; WEF, 2000).

Para o preparo das soluções de alimentação das colunas de leito fixo, as amostras de água foram fortificadas com 2,4-D na concentração teórica inicial de $7 \mathrm{mg} . \mathrm{L}^{-1}$. As soluções foram filtradas em membrana de fibra de vidro com $0,45 \mu \mathrm{m}$ de porosidade, conforme orientação da norma ASTM D 6586-03 (ASTM, 2008). O pH foi ajustado para 6,5 com solução aquosa de hidróxido de sódio $\left(0,1\right.$ mol.L $\left.\mathrm{L}^{-1}\right)$.

\section{Preparo dos padrões, amostragem e procedimento analítico para determinação da concentração de 2,4-D}

Foi preparada solução estoque de 2,4-D (Sigma Aldrich, Estados Unidos) em acetonitrila (J.T. Baker, Estados Unidos) na concentração de $0,1 \mathrm{~g} \cdot \mathrm{L}^{-1}$. O solvente foi previamente filtrado em membrana filtrante para solvente orgânico (porosidade de $0,22 \mu \mathrm{m}$, Sartorius) e a solução foi mantida em frascos âmbar a $4^{\circ} \mathrm{C}$.

As análises para quantificação do 2,4-D foram realizadas em cromatógrafo líquido da marca Shimadzu CBM-20A, com desgaseificador DGU-20AS, bombas LC-20AT, injetor automático SIL-20AHT, forno CTO-20A e detector de arranjo de diodos (DAD) SPD-M20A com o espectro de absorção em uma faixa de 190 a $300 \mathrm{~nm}$. Utilizou-se uma coluna C-18 (Lichrospher $100 \mathrm{RP}-18,5 \mu \mathrm{m}, 250 \times 4 \mathrm{~mm}$ ) acoplada a uma pré-coluna (Lichrospher $100 \mathrm{RP}-18,5 \mu \mathrm{m}, 4 \times 4 \mathrm{~mm}$ ), em condições isocráticas com fase móvel constituída por mistura $\left(42: 58\right.$ v.v $\left.{ }^{-1}\right)$ de acetonitrila/água (acidificada com $\mathrm{H}_{3} \mathrm{PO}_{4}-\mathrm{pH}=3$,2) com vazão de $0,8 \mathrm{~mL} \cdot \mathrm{min}^{-1}$. A temperatura do forno da coluna foi fixada em $30^{\circ} \mathrm{C}$ e o volume de injeção da amostra foi de $20 \mu \mathrm{L}$. O comprimento de onda utilizado para identificação e quantificação do 2,4-D, por calibração externa, foi de $200 \mathrm{~nm}$. Os dados foram processados utilizando o software LCSolutions (versão 2.1).

A etapa de extração em fase sólida (EFS) foi adaptada do método proposto por Faria (2004). Os parâmetros avaliados para a validação do método de EFS por cromatografia líquida de alta eficiência com detector de arranjo de fotodiodo (EFS-CLAE-DAD) foram: linearidade, limite de detecção (LD), limite de quantificação (LQ); recuperação e precisão foram determinados de acordo com a Resolução n 899/2003 da ANVISA (BRASIL, 2003b).

\section{Sistema adsortivo em escala reduzida}

Para a determinação dos parâmetros a serem utilizados em sistemas adsortivos de leitos fixos em escala reduzida, foram utilizados dados obtidos a partir de sistemas adsortivos em escala real e as equações sugeridas por Crittenden et al. (1987).

O cálculo do tempo de contato em leito vazio (TCLV) para coluna em escala reduzida foi realizado de acordo com a Equação 1.

$\frac{\operatorname{TCLV}_{\text {reduzida }}}{\operatorname{TCLV}_{\text {real }}}=\left[\frac{\mathrm{d}_{\text {reduzida }}}{\mathrm{d}_{\text {real }}}\right]^{2-\mathrm{x}}$

Em que:

$\mathrm{TCLV}_{\text {reduzida }}=$ tempo de contato em leito vazio para coluna em escala reduzida $(\mathrm{min})$;

$\mathrm{TCLV}_{\text {real }}=$ tempo de contato em leito vazio para coluna em escala real (min);

$\mathrm{d}_{\text {reduzida }}=$ diâmetro médio da partícula de CAG usada na coluna em escala reduzida $(\mathrm{mm})$;

$\mathrm{d}_{\text {real }}=$ diâmetro médio da partícula de CAG usada na coluna em escala real (mm);

$\mathrm{x}=$ fator de difusividade intrapartícula, sendo igual a 0 para difusividade constante, e igual a 1 para difusividade proporcional.

Em relação à determinação do valor para o fator de difusividade intrapartícula, dois conceitos devem ser lembrados: o da difusividade constante (DC), que assume que o coeficiente de difusão do adsorvato é independente do tamanho da partícula de CAG; e o da difusividade proporcional (DP), que assume que o coeficiente de difusão é linearmente dependente do tamanho da partícula de CAG (CRITTENDEN et al., 1987). Segundo Snoeyink e Summers (1999), para a DC, as vazões variam de 50 a $150 \mathrm{~mL} . \mathrm{min}^{-1}$; e para a DP as vazões variam de 5 a $20 \mathrm{~mL} \cdot \mathrm{min}^{-1}$. 
O cálculo da taxa de aplicação superficial para coluna em escala reduzida foi realizado conforme a Equação 2.

$\mathrm{TAS}_{\text {reduzida }}=\mathrm{TAS}_{\text {real }} \cdot \frac{\mathrm{d}_{\text {real }}}{\mathrm{d}_{\text {reduzida }}} \cdot \frac{\operatorname{Re}_{\text {reduzida }}}{\operatorname{Re}_{\text {real }}}$

Em que:

$\mathrm{TAS}_{\text {reduzida }}=$ taxa de aplicação superficial para coluna em escala reduzida $\left(\mathrm{m} \cdot \mathrm{h}^{-1}\right)$;

TAS $_{\text {real }}=$ taxa de aplicação superficial para a coluna em escala real $\left(\mathrm{m} \cdot \mathrm{h}^{-1}\right)$; $\mathrm{d}_{\text {real }}=$ diâmetro médio da partícula de CAG usada na coluna em escala real $(\mathrm{mm})$;

$\mathrm{d}_{\text {reduzida }}=$ diâmetro médio da partícula de CAG usada na coluna em escala reduzida $(\mathrm{mm})$;

$\mathrm{Re}_{\text {reduzida }}=$ número de Reynolds da coluna em escala reduzida;

$\mathrm{Re}_{\text {real }}=$ número de Reynolds da coluna em escala real.

No dimensionamento de projetos segundo a DC, considera-se que os valores do número de Reynolds em escala real e reduzida são iguais (MWH, 2005). Já no dimensionamento segundo a DP, o valor do número de Reynolds para coluna em escala real pode ser determinado através da Equação 3.

$R e_{\text {real }}=\frac{\rho v d}{\mu}$

Na qual:

$\mathrm{Re}_{\text {real }}=$ número de Reynolds da coluna em escala real;

$\rho=$ densidade do fluido $\left(\mathrm{kg} . \mathrm{m}^{-3}\right)$;

$\mathrm{v}=$ velocidade intersticial $\left(\mathrm{m} . \mathrm{s}^{-1}\right)$;

$\mathrm{d}=$ diâmetro médio da partícula de CAG $(\mathrm{m})$;

$\mu=$ viscosidade dinâmica do fluido (Pa.s ou kg. $\mathrm{m}^{-1} \cdot \mathrm{s}^{-1}$ ).

A velocidade intersticial, por sua vez, pode ser calculada através da Equação 4.

$\mathrm{v}=\frac{\text { TAS }_{\text {real }}}{\varepsilon}$

Em que:

$\mathrm{TAS}_{\text {real }}=$ taxa de aplicação superficial para a coluna em escala real $\left(\mathrm{m} \cdot \mathrm{h}^{-1}\right)$; $\varepsilon=$ porosidade do leito (adimensional).

Em relação ao valor do número de Reynolds para coluna em escala reduzida, Crittenden et al. (1987) sugerem a escolha de um valor mínimo, que denominaram de "número de Reynolds mínimo" $\left(\mathrm{Re}_{\min }\right)$, entre 0,5 e 1 . Porém ainda não há, na literatura, informações definidas acerca dos critérios necessários para se estabelecer o valor do $\mathrm{Re}_{\min }$.

O cálculo da altura do leito para coluna em escala reduzida foi realizado utilizando a Equação 5.
$\mathrm{H}_{\text {reduzida }}=\mathrm{TAS}_{\text {reduzida }} \cdot \mathrm{TCLV}_{\text {reduzida }}$

Na qual:

$\mathrm{H}_{\text {reduzida }}=$ altura do leito $(\mathrm{cm})$;

TAS $_{\text {reduzida }}=$ taxa de aplicação superficial para coluna em escala reduzida $\left(\mathrm{m} \cdot \mathrm{h}^{-1}\right)$;

$\mathrm{TCLV}_{\text {reduzida }}=$ tempo de contato em leito vazio para coluna em escala reduzida (min).

O cálculo da vazão do sistema adsortivo em escala reduzida foi realizado conforme a Equação 6.

$\mathrm{Q}_{\text {reduzida }}=\mathrm{TAS}_{\text {reduzida }} \cdot \mathrm{A}_{\text {reduzida }}$

Na qual:

$\mathrm{Q}_{\text {reduzida }}=$ vazão $\left(\mathrm{em} \mathrm{mL} \cdot \mathrm{min}^{-1}\right)$;

$\mathrm{TAS}_{\text {reduzida }}=$ taxa de aplicação superficial para coluna em escala reduzida $\left(\mathrm{m} \cdot \mathrm{h}^{-1}\right)$;

$\mathrm{A}_{\text {reduzida }}=$ área da seção circular da coluna em escala reduzida $\left(\mathrm{cm}^{2}\right)$.

Na determinação dos parâmetros do sistema adsortivo em escala reduzida foram utilizadas as Equações de 1 a 6, para difusividade proporcional, visto que a vazão estava na faixa de 5 a $20 \mathrm{~mL}$. $\mathrm{min}^{-1}$, conforme orientações de Snoeyink e Summers (1999). Os demais parâmetros foram definidos considerando a existência de um leito adsorvente já existente em escala piloto e as recomendações da ASTM D 6586-03 (ASTM, 2008). Para a taxa de aplicação superficial, foi adotado o valor de $120 \mathrm{~m}^{3} \cdot \mathrm{m}^{-2} \cdot \mathrm{dia}^{-1}$ e para o TCLV, o tempo de 5 minutos. MWH (2005) sugere que a taxa de aplicação em leitos adsorventes esteja na faixa de $120 \mathrm{a} 360 \mathrm{~m}^{3} \cdot \mathrm{m}^{-2} \cdot \mathrm{dia}^{-1} \mathrm{e}$ o TCLV varie entre 5 e 30 minutos. Os valores encontram-se na Tabela 1.

Tabela 1 - Parâmetros de dimensionamento das colunas em escala reduzida e dados de escala real.

\begin{tabular}{|c|c|c|c|}
\hline Parâmetros do projeto & Unidade & $\begin{array}{c}\text { Escala } \\
\text { real }\end{array}$ & $\begin{array}{l}\text { Escala } \\
\text { reduzida }\end{array}$ \\
\hline \multirow{2}{*}{ Granulometria do carvão } & Mesh & $8 \times 30$ & $60 \times 80$ \\
\hline & $\mathrm{mm}$ & $0,59-2,38$ & $0,177-0,250$ \\
\hline Diâmetro médio da partícula & $\mathrm{mm}$ & 1,485 & 0,214 \\
\hline Densidade aparente & g.cm ${ }^{-3}$ & 0,59 & 0,63 \\
\hline Tempo de contato em leito vazio & $\min$ & 5 & 0,72 \\
\hline \multirow{2}{*}{ Taxa de aplicação superficial } & $m \cdot h^{-1}$ & 5 & 5 \\
\hline & $\mathrm{m}^{3} \cdot \mathrm{m}^{-2} \cdot \mathrm{dia}^{-1}$ & 120 & 120 \\
\hline Vazão & $\mathrm{mL} \cdot \mathrm{min}^{-1}$ & - & 6,5 \\
\hline Número de Reynolds & adimensional & 3,61 & 0,55 \\
\hline Diâmetro da coluna & $\mathrm{cm}$ & - & 1 \\
\hline Altura do leito & $\mathrm{cm}$ & - & 6,0 \\
\hline Volume do leito & $\mathrm{cm}^{3}$ & - & 4,71 \\
\hline
\end{tabular}


No sistema, utilizaram-se duas colunas de adsorção em série com $1,0 \mathrm{~cm}$ de diâmetro interno (buretas em vidro borossilicato com capacidade de $50 \mathrm{~mL}$ ). A solução de alimentação foi armazenada em barrilhete de PVC com capacidade de $10 \mathrm{~L}$. As vazões foram controladas por meio de bombas dosadoras peristálticas (modelos BPM 301 e 302, Allifer). Os ensaios de adsorção foram executados em fluxo contínuo com descarga livre, conforme instalação experimental esquematizada na Figura 2.

No preparo do carvão, seguiram-se as recomendações das normas ASTM D 6586-03 e ASTM D 3922-89 (ASTM, 1989). O leito de carvão foi apoiado em uma tela protegida por uma fina camada de lã de vidro, com o objetivo de evitar a passagem do adsorvente. O carvão molhado foi colocado cuidadosamente dentro das colunas, mantendo-se, durante a transferência, uma camada de água ultrapura acima do adsorvente. No topo da camada de carvão ativado, colocou-se uma fina camada de lã de vidro e algumas pérolas de vidro para evitar a flutuação de partículas e permitir a distribuição uniforme da solução de alimentação.

Após a montagem, as colunas foram alimentadas com água ultrapura em um fluxo ascendente com vazão de 2,0 mL.min ${ }^{-1}$ para que as partículas do carvão se acomodassem e a presença de ar no leito fosse minimizada. A seguir, deixou-se passar água ultrapura em fluxo descendente na vazão estabelecida para o ensaio até a estabilização do sistema. Após a estabilização, as colunas foram alimentadas com as soluções de alimentação fortificadas com o 2,4-D.

As amostras foram coletadas em três pontos: entrada da primeira coluna e saídas da primeira e da segunda coluna. As primeiras coletas foram realizadas entre 60 e 90 minutos após o início dos ensaios,

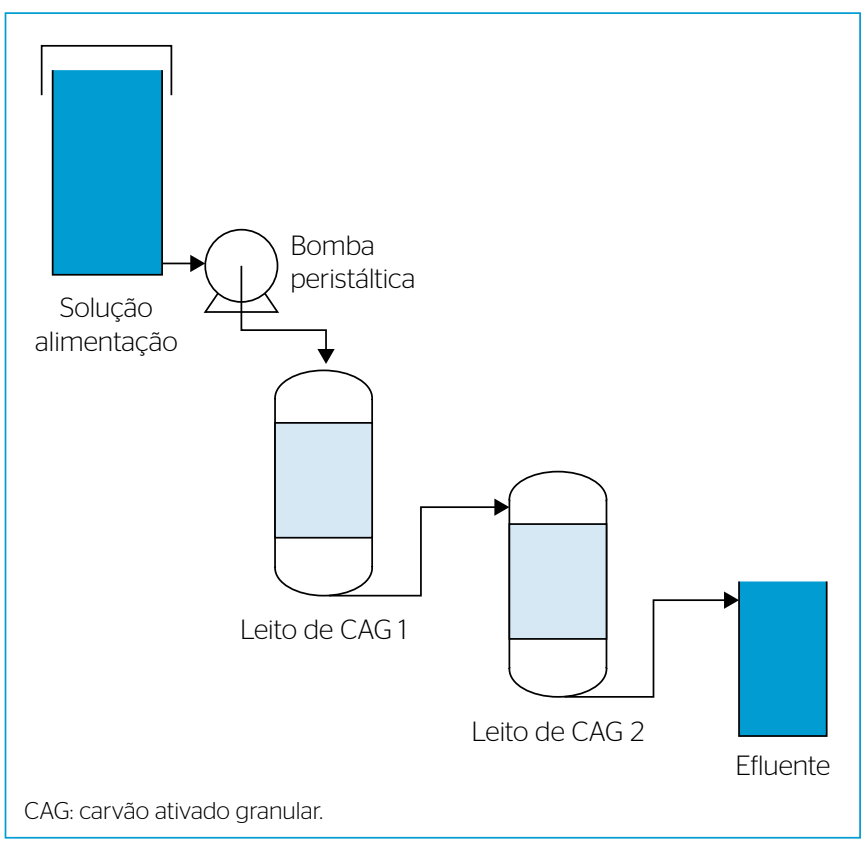

Figura 2 - Esquema da instalação experimental para os ensaios de adsorção do ácido 2,4-diclorofenoxiacético. e as demais, a cada 2 horas em frascos âmbar de $100 \mathrm{~mL}$. As amostras coletadas foram armazenadas em geladeira.

O ponto de ruptura foi definido como o ponto no qual foi alcançada a concentração máxima permitida para o 2,4-D, segundo a Portaria MS no 2914 (BRASIL, 2011), que é de $30 \mu \mathrm{g} . \mathrm{L}^{-1}$. As curvas de ruptura do 2,4-D foram construídas a partir dos dados obtidos nos ensaios de adsorção em amostras de água ultrapura e de água filtrada proveniente de ETA. Essas curvas foram expressas pela concentração de 2,4-D (em $\mu \mathrm{g} . \mathrm{L}^{-1}$ ) em função do volume de leito tratado (volume de água tratada em relação ao volume do leito de CAG).

Para a determinação da capacidade de adsorção $\left(\mathrm{q}_{\mathrm{R}}\right)$, utilizou-se a Equação 7 (MHW, 2005).

$\mathrm{q}_{\mathrm{R}}=\frac{\mathrm{V}}{\mathrm{M}}\left(\mathrm{C}_{0}-\mathrm{C}_{\mathrm{R}}\right)$

Na qual:

$\mathrm{q}_{\mathrm{R}}=$ capacidade de adsorção no ponto de ruptura (mg de adsorvato/g de adsorvente);

$\mathrm{V}=$ volume da solução $(\mathrm{L})$;

$\mathrm{M}=$ massa do adsorvente $(\mathrm{g})$;

$\mathrm{C}_{0}=$ concentração inicial do adsorvato em fase aquosa (mg. $\left.\mathrm{L}^{-1}\right)$;

$\mathrm{C}_{\mathrm{R}}=$ concentração do adsorvato em fase aquosa no ponto de ruptura $\left(\mathrm{mg} \cdot \mathrm{L}^{-1}\right)$.

\section{RESULTADOS E DISCUSSÃO}

\section{Caracterização do carvão ativado granular e das amostras de água}

As principais características apresentadas pelo CAG foram: densidade aparente $0,63 \mathrm{~g} \cdot \mathrm{cm}^{-3}$, ASE de $530 \mathrm{~m}^{-2} \cdot \mathrm{g}^{-1}$, volume total de microporos de $0,26 \mathrm{~cm}^{3} \cdot \mathrm{g}^{-1}$ com dimensão mínima de $6,14 \AA$, volume de mesoporos de $0,0471 \mathrm{~cm}^{3} \cdot \mathrm{g}^{-1}$ com dimensão máxima de $344,2 \AA$, número de iodo de $598 \mathrm{mg}$ de iodo.g-1 e o pH da água de lavagem igual a 9,19.

A American Water Works Association (AWWA, 2005) recomenda que os carvões utilizados em tratamento de água possuam área superficial variando de 650 a $1.000 \mathrm{~m}^{2} \cdot \mathrm{g}^{-1}$. Contudo, Droste (1997) afirma que para os carvões ativados comerciais, esse valor varia de $500 \mathrm{a} 1.600 \mathrm{~m}^{2} . \mathrm{g}^{-1}$.

De acordo com os resultados de caracterização, o carvão foi classificado como predominantemente microporoso, segundo os critérios da International Union of Pure and Applied Chemistry (IUPAC) (BANSAL; GOYAL, 2005). Os microporos proporcionam alta capacidade de adsorção de moléculas de pequenas dimensões, tais como o 2,4-D, que possui largura de 2,074 Å. Em relação ao volume de microporos, o valor obtido encontra-se dentro da faixa sugerida por Bansal e Goyal (2005), que é de 0,15 a $0,70 \mathrm{~cm}^{3} \cdot \mathrm{g}^{-1}$. Em relação ao número de iodo e à densidade aparente, verificou-se que os valores obtidos estão 
de acordo os valores mínimos sugeridos pela AWWA (2005) para carvões a serem utilizados em ETAs, superiores a 500 e 0,25 g.cm ${ }^{-3}$, respectivamente. A análise do infravermelho apresentou bandas na região de 1081 a $1085 \mathrm{~cm}^{-1}$, que correspondem a grupamentos alcóolicos e fenólicos, justificando o $\mathrm{pH}$ básico de 9,19 encontrado para o carvão (BANSAL; GOYAL, 2005; SILVERSTEIN; WEBSTER; KIEMLE, 2010).

Os ensaios foram realizados com dois tipos de água fortificadas com 2,4-D em concentração aproximada de $7.089 \pm 104 \mu \mathrm{g} . \mathrm{L}^{-1}$. As características das amostras de águas utilizadas para o preparo das soluções de alimentação encontram-se na Tabela 2.

\section{Quantificação do 2,4-D}

As análises para quantificação foram realizadas em cromatógrafo líquido e foi necessária uma etapa de extração em fase sólida para quantificação do 2,4-D. Os resultados encontrados para validação do método estão apresentados na Tabela 3.

Tabela 2 - Características físico-químicas das amostras de água de alimentação.

\begin{tabular}{l|c|c|c} 
Parâmetros & Unidades & $\begin{array}{c}\text { Água } \\
\text { ultrapura }\end{array}$ & $\begin{array}{c}\text { Água filtrada } \\
\text { mín.-máx. }\end{array}$ \\
\hline $\mathrm{pH}$ & adimensional & 6,8 & $5,6-6,7$ \\
\hline Temperatura & ${ }^{\circ} \mathrm{C}$ & $22 \pm 1$ & $20-22$ \\
\hline Turbidez & $\mathrm{UT}$ & $\leq 0,5$ & $0,56-0,64$ \\
\hline Cor verdadeira & $\mathrm{uH}$ & $\leq 2,0$ & $3-14$ \\
\hline Alcalinidade & $\mathrm{mg} \mathrm{de} \mathrm{CaCO}_{3} \mathrm{~L}^{-1}$ & - & $10,3-20,9$ \\
\hline Condutividade & $\mu \mathrm{SSCm}^{-1}$ & - & $55,6-58,4$ \\
\hline Absorbância UV-254 & adimensional & ALD & 0,018-0,025 \\
\hline Ácido & $\mu \mathrm{Mg.L}^{-1}$ & ALD & ALD \\
\hline 2,4-diclorofenoxiacético & & & \\
\hline
\end{tabular}

ALD: abaixo do limite de detecção do método de extração em fase sólida por cromatografia líquida de alta eficiência com detector de arranjo de fotodiodo (EFS-CLAE-DAD).

Tabela 3 - Dados de validação para a quantificação do ácido 2,4-diclorofenoxiacético.

\begin{tabular}{l|c} 
Parâmetros de validação & $30-10.000$ \\
\hline Linearidade $\left(\mu \mathrm{g} \cdot \mathrm{L}^{-1}\right)$ & 10,8 \\
\hline Limite de detecção $\left(\mu \mathrm{g} \cdot \mathrm{L}^{-1}\right)$ - detector DAD & 36,0 \\
\hline Limite de quantificação $\left(\mu \mathrm{g} \mathrm{L}^{-1}\right)$ - detector DAD & $1,46-2,84$ \\
\hline Precisão (\%) & 102 \\
\hline Método EFS-CLAE-DAD & 0,61 \\
\hline Recuperação em água destilada e deionizada (\%) & 2,0 \\
\hline Limite de detecção $\left(\mu g \cdot \mathrm{L}^{-1}\right)$ - água filtrada de ETA & 110 \\
\hline Limite de quantificação $\left(\mu \mathrm{g} . \mathrm{L}^{-1}\right)$-água filtrada de ETA &
\end{tabular}

DAD: detector de arranjo de fotodiodo; EFS-CLAE-DAD: extração em fase sólida por cromatografia líquida de alta eficiência com detector de arranjo de fotodiodo:

ETA: estação de tratamento de água.

\section{Resultados dos ensaios de remoção de 2,4-D}

Foram realizados dois ensaios com adição de 2,4-D nas amostras afluentes às colunas de adsorção. Um ensaio com amostra de água ultrapura e outro ensaio com água filtrada proveniente de ETA. Na Tabela 4, encontram-se as condições experimentais utilizadas. Considerando o limite do 2,4-D, definido pela Portaria MS nº 2.914 (BRASIL, 2011), o ponto de ruptura foi definido para a condição em que a concentração do agrotóxico alcançou o valor de $30 \mu \mathrm{g}$. $\mathrm{L}^{-1}$. A capacidade de adsorção no ponto de ruptura foi avaliada utilizando a Equação 7. A condição de ruptura do leito foi avaliada também pela relação entre a concentração afluente $\left(\mathrm{C}_{0}\right)$ e a concentração efluente $(\mathrm{C})$ representada por $\mathrm{C} / \mathrm{C}_{0}$. Todos os ensaios foram encerrados considerando as condições de ruptura de $30 \mu \mathrm{g} . \mathrm{L}^{-1}$ e alcance da perda de carga máxima no primeiro leito sem retrolavagem.

O período total do ensaio em água ultrapura foi de 1.915 minutos (31 horas e 55 minutos), sendo esse tempo suficiente para alcançar a concentração de ruptura ( $\left.30 \mu \mathrm{g} . \mathrm{L}^{-1}\right)$ no primeiro leito. O ensaio foi encerrado pelo alcance da perda de carga sem que o segundo leito alcançasse a ruptura. A ruptura no primeiro leito ocorreu em $1.349 \mathrm{minu}-$ tos (22 horas e 29 minutos) para volume de água tratada por volume de CAG igual a 2.090 e $\mathrm{C} / \mathrm{C}_{0}$ igual a 0,004 . O primeiro leito adsorveu, até o ponto de ruptura, 22,0 mg de 2,4-D por grama de CAG; e a concentração de 2,4-D no efluente do segundo leito foi $1,38 \mu \mathrm{g} \cdot \mathrm{L}^{-1}$. Salman, Njoku e Hameed (2011) estudaram a adsorção do 2,4-D em carvão ativado proveniente de palmeira com concentração de 2,4-D de $50 \mathrm{mg} \cdot \mathrm{L}^{-1} \mathrm{em}$ microcolunas, vazão de $10 \mathrm{~mL} \cdot \mathrm{min}^{-1} \mathrm{e}$ altura de leito de $3 \mathrm{~cm}$. Nessas condições, a capacidade de adsorção foi de $15,1 \mathrm{mg}$ de 2,4-D por grama de CAG, com tempo de 270 minutos na condição de ruptura do leito em $\mathrm{C} / \mathrm{C}_{0}$ igual a 0,05 . A curva de ruptura para o ensaio em água ultrapura está representada na Figura 3.

Tabela 4 - Parâmetros experimentais dos ensaios.

\begin{tabular}{l|c|c|c|c}
\multirow{2}{*}{ Parâmetros } & \multicolumn{2}{|c|}{ Água ultrapura } & \multicolumn{2}{c}{ Água filtrada } \\
\cline { 2 - 5 } & coluna 1 & coluna 2 & coluna 1 & coluna 2 \\
\hline TCLV (min) & 0,70 & 0,70 & 0,74 & 0,74 \\
\hline Vazão média (mL.min'1) & $6,8 \pm 0,7$ & $6,8 \pm 0,7$ & $6,3 \pm 0,4$ & $6,3 \pm 0,4$ \\
\hline $\begin{array}{l}\text { Taxa de aplicação } \\
\text { superficial (m.h-1) }\end{array}$ & 5,0 & 5,0 & 5,0 & 5,0 \\
\hline Diâmetro da coluna (cm) & 1,0 & 1,0 & 1,0 & 1,0 \\
\hline Altura do leito (cm) & 5,6 & 5,5 & 5,2 & 5,5 \\
\hline Massa de CAG (g) & 2,9455 & 2,9456 & 2,9456 & 2,9455 \\
\hline Densidade do leito (g.cm $\left.{ }^{-3}\right)$ & 0,67 & 0,68 & 0,72 & 0,68 \\
\hline $\begin{array}{l}\text { Ácido } \\
\text { 2,4-diclorofenoxiacético } \\
\text { no afluente ( } \mu \text { g.L-1) }\end{array}$ & $7.089 \pm 104$ & - & $7.029 \pm 96$ & - \\
\hline \begin{tabular}{l} 
Duração do ensaio (min) \\
\hline
\end{tabular} & 1.964 & 2009 & - & - \\
\hline
\end{tabular}

TCLV: tempo de contato do leito em vazio; CAG: carvão ativado granular. 
Com relação aos mecanismos que governam a adsorção do 2,4-D no carvão ativado, ainda existem lacunas a serem preenchidas, pois para $\mathrm{pH}$ acima do pKa de 2,8 do 2,4-D, as moléculas estão carregadas negativamente, bem como a superfície do carvão ativado, indicando que outras variáveis não quantificadas por ligações eletrostáticas atuam na adsorção do 2,4-D. Resultados semelhantes foram encontrados por Aksu e Kabasakal (2004).

Considerando que a capacidade de adsorção em leito fixo depende das características do carvão ativado, das características do afluente, do $\mathrm{pH}$ da fase líquida, das características hidráulicas e físicas do sistema e da concentração do composto no afluente, os resultados apontam o CAG utilizado como promissor adsorvente a ser utilizado em sistemas de tratamento de água para abastecimento.

A relevância do ensaio em água natural filtrada diz respeito à consideração da presença de matéria orgânica em águas naturais e à influência que ela causa na capacidade adsortiva do 2,4-D. A curva de ruptura para o ensaio em água natural filtrada está representada na Figura 4.

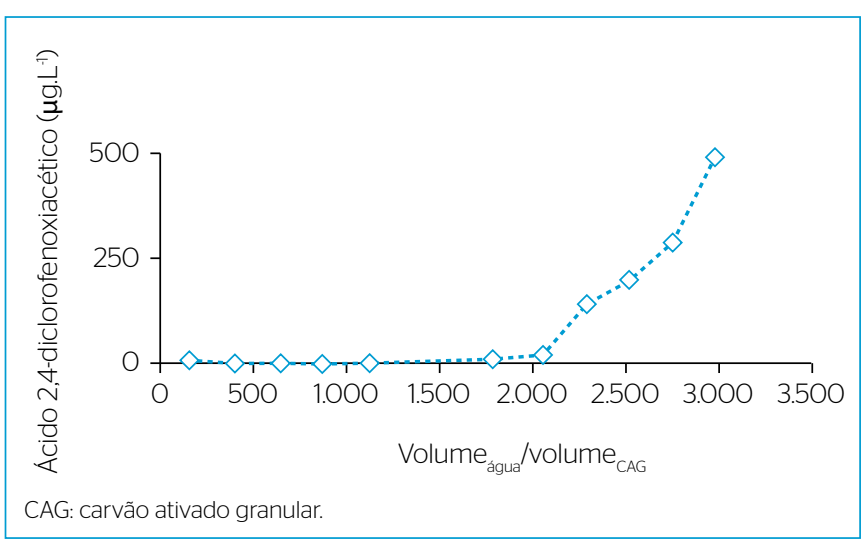

Figura 3 - Curva de ruptura do ácido 2,4-diclorofenoxiacético do primeiro leito obtida para o ensaio 1 (água ultrapura fortificada). Condições do ensaio: vazão de $(6,7 \pm 0,7) \mathrm{mL} \mathrm{min}^{-1}$; taxa de aplicação superficial de $5,0 \mathrm{~m} \cdot \mathrm{h}^{-1}$; temperatura ambiente $\left(22 \pm 10^{\circ} \mathrm{C}\right)$; $\mathrm{pH}(6,8)$; $[2,4-D]=(7.089 \pm 104) \mu \mathrm{g} \cdot \mathrm{L}^{-1}$.

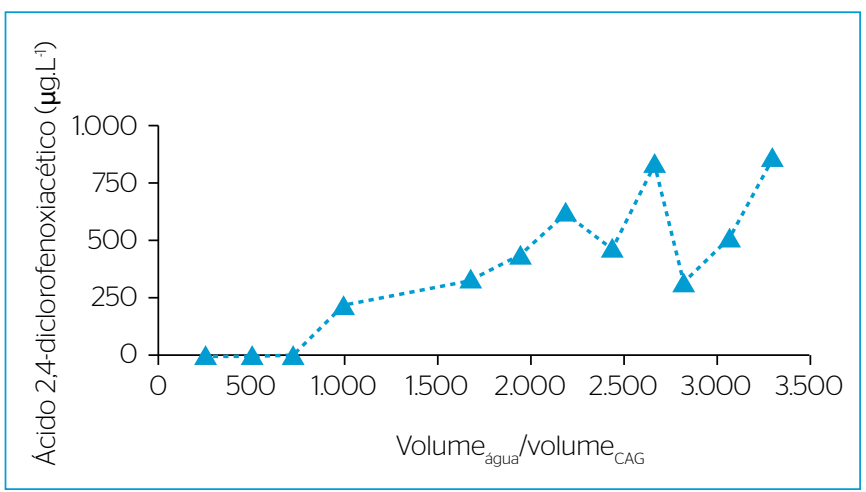

Figura 4-Curva de ruptura doácido2,4-diclorofenoxiacético do primeiro leito obtida para o ensaio 2 (água filtrada de estação de tratamento de água fortificada). Condições do ensaio: vazão de $(6,3 \pm 0,4) \mathrm{mL} \cdot \mathrm{min}^{-1}$; taxa de aplicação superficial de $5,0 \mathrm{~m} \cdot \mathrm{h}^{-1}$; temperatura $\left(21 \pm 1^{\circ} \mathrm{C}\right)$; $\mathrm{pH}(5,6-6,7)$; $[2,4-D]=(7.029 \pm 96) \mu \mathrm{g} \cdot \mathrm{L}^{-1}$.
No ensaio realizado com água filtrada de ETA, o ponto de ruptura do primeiro leito foi atingido no tempo de 567 minutos ( 9 horas e 27 minutos), para valor de volume de água/volume de CAG igual a 766, correspondendo à capacidade de adsorção de 7,4 mg de 2,4-D por grama de $\mathrm{CAG}$, com valor de $\mathrm{C} / \mathrm{C}_{0}$ igual 0,004. No tempo de 567 minutos, a matéria orgânica, avaliada pela absorvância, foi adsorvida pelo carvão ativado na relação de $\mathrm{C} / \mathrm{C}_{0}$ igual a 0,24 . O ensaio 2 foi finalizado antes que o ponto de ruptura fosse alcançado no segundo leito, com duração total de 1.964 minutos (32 horas e 44 minutos), sendo encerrado pela perda de carga no leito 1 . Todas as amostras coletadas na saída do segundo leito apresentaram concentrações inferiores ao limite de quantificação do método analítico, ou seja, abaixo de 2,0 $\mu \mathrm{g} \cdot \mathrm{L}^{-1}$.

A água filtrada proveniente de ETA possuía uma fração de MON que estava presente na água bruta, avaliada pelos parâmetros cor e absorvância, e que não foi removida após os processos de coagulação e filtração. $\mathrm{O}$ tamanho molecular dos ácidos húmicos é equivalente a uma esfera de 60 a $100 \AA ̊$ e dos ácidos fúlvicos, a uma esfera de 20 a $30 \AA$ A. Na coagulação, são removidas 58\% das moléculas de MON de tamanho maior; e a fração restante tem acesso ao CAG (EBIE et al., 2001). As moléculas menores podem ocupar a região microporosa do CAG através da competição direta pelos sítios do carvão, e as maiores podem interferir no bloqueio dos poros do carvão (MATSUI et al., 2002; JARVIE et al., 2005).

\section{Comparação entre os ensaios com água ultrapura e água filtrada}

$\mathrm{Na}$ Figura 5, encontram-se as curvas de ruptura do 2,4-D dos ensaios com água ultrapura e com água natural e dados referentes à absorvância e condutividade para o primeiro leito. As curvas de ruptura foram representadas na ordenada pela relação entre $\mathrm{a}_{0}$ e $\mathrm{C}$ para discussão dos resultados considerando a presença de matéria orgânica.

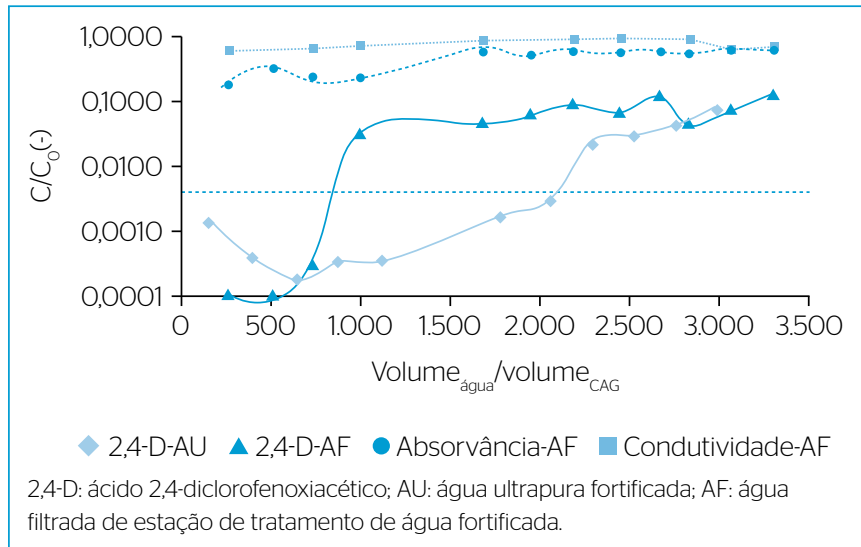

Figura 5 - Curvas de ruptura do ácido 2,4-diclorofenoxiacético, absorvância e condutividade para o primeiro leito dos ensaios 1 e 2 . A linha pontilhada indica o valor de $30 \mu \mathrm{g} \cdot \mathrm{L}^{-1}$. 
Para a condição definida por volume $e_{\text {agua }}$ volume $_{\text {CAG }}$ igual a 1.000, com o carvão virgem, ocorreu maior adsorção do 2,4-D e da matéria orgânica avaliada como absorvância. A partir dessa condição, a taxa de adsorção diminui e tende a se tornar constante ao longo do tempo. Dehghani, Nasseri e Karamimanesh (2014) encontraram resultados semelhantes quando estudaram a influência do tempo, em ensaio de laboratório, na remoção de 2,4-D em CAG. O fenômeno foi explicado pela presença de grande quantidade de sítios disponíveis na superfície do carvão, contribuindo para a alta taxa de adsorção. Com o tempo, os sítios vão tornando-se indisponíveis e a taxa de adsorção é controlada pela taxa de migração do contaminante para canais mais profundos dos poros.

Comparando-se as curvas de ruptura para o primeiro leito, apresentadas na Figura 5, é possível verificar a influência da matéria orgânica presente na água filtrada da ETA. A tendência à saturação do carvão ocorre primeiro para a matéria orgânica avaliada como absorvância para a condição de $\mathrm{C} / \mathrm{C}_{0}$ igual a 0,2 e relação $\mathrm{V}_{\text {água }} / \mathrm{V}_{\mathrm{CAG}}$ superior a 733. Essa condição foi interpretada como responsável pela redução da capacidade de adsorção do 2,4-D em 67\%, quando comparado com o ensaio realizado em amostra de água ultrapura fortificada com 2,4-D.

As moléculas de MON e de 2,4-D possuem tamanhos diferentes e ocupam sítios de adsorção também diferentes. As moléculas de MON ocupam os sítios de tamanhos maiores, tornando-os indisponíveis para o acesso das moléculas do 2,4-D, diminuindo a competição e sendo mais importante o fenômeno de bloqueio dos microporos ao longo do tempo. Os íons dissolvidos avaliados pela condutividade tiveram pequena fração adsorvida $\left(\mathrm{C} / \mathrm{C}_{0}\right.$ máximo de 0,61$)$, não comprometendo a adsorção do 2,4-D.

Para a adsorção do 2,4-D a partir da relação volume $e_{\text {agua }} /$ volume $_{\mathrm{CAG}}$ superior a 2.750, iniciou-se uma aproximação das curvas de adsorção em água ultrapura e água filtrada com valores de $\mathrm{C} / \mathrm{C}_{0}$ próximos a 0,04 . Essa condição não foi observada nos trabalhos experimentais de outros pesquisadores.

Apesar de a presença da MON ter interferido na capacidade de adsorção do 2,4-D, foi possível avaliar a capacidade de adsorção, em águas naturais, utilizando os testes adsortivos em microcolunas. Segundo Ersan et al. (2016), a capacidade de adsorção de contaminantes orgânicos em CAG em águas com presença de MON depende mais das propriedades físico-químicas e estruturais dos microcontaminantes e das características do adsorvato do que do tipo de MON; e os resultados obtidos no presente trabalho podem ser utilizados como referência para estudos iniciais de remoção de 2,4-D em ETAs para o material adsorvente utilizado. O CAG utilizado possui a capacidade de adsorver o 2,4-D em águas com presença de matéria orgânica para valor de volume de água/volume de CAG igual a 766, com capacidade de adsorção de 7,4 mg de 2,4-D por grama de CAG, produzindo efluente com concentração de $30 \mu \mathrm{g}$. $\mathrm{L}^{-1}$, limite máximo permitido na Portaria MS no 2.914/2011 (BRASIL, 2011).

\section{CONCLUSÕES}

O uso de sistemas RSSCT permitiu prever o desempenho do CAG para remoção do 2,4-D em águas com diferentes composições de MON e estimar o tempo de ruptura e a relação $\mathrm{V}_{\text {água }} / \mathrm{V}_{\mathrm{CAG}}$ para cada condição.

Ao se comparar os ensaios com água ultrapura e água filtrada proveniente de ETA, verificou-se que a presença de MON reduziu a capacidade de adsorção do 2,4-D pelo CAG em $66 \%$ para produção de efluente com concentração de 2,4-D inferior a $30 \mu$ g.L $L^{-1}$. Para essa condição de ruptura, a capacidade de adsorção foi de 7,43 mg de 2,4-D por grama de $C A G$, sendo para a relação o $V_{\text {agua }} / V_{C A G}$ estimado em 766 .

O CAG mostrou-se efetivo para remoção do 2,4-D e o resultado poderá ser utilizado como avaliação inicial da capacidade de adsorção de leitos adsorventes para remoção do 2,4-D em águas naturais com a presença de matéria orgânica que apresente características semelhantes.

\section{FONTE DE FINANCIAMENTO}

Financiadora de Estudos e Projetos (Finep) e Universidade Federal do Espírito Santo.

\section{REFERÊNCIAS}

AKSU,Z:KABASAKAL,E.(2004)Batchadsorptionof2,4-dichlorophenoxyacetic acid (2,4-D) from aqueous solution by granular activated carbon. Separation and Purification Technology, v. 35, p. 223-240.

AMERICAN PUBLIC HEALTH ASSOCIATION (APHA); AMERICAN WATER WORKS ASSOCIATION (AWWA); WATER ENVIRONMENTAL FEDERATION (WEF). (2000) Standard Methods for the Examination of Water and Wastewater. 20. ed. Washington, D.C.: APHA.
AMERICAN SOCIETY FOR TESTING AND MATERIALS (ASTM). (1989) ASTM D 3922-89: Standard Practice for Estimating the Operating Performance of Granular Activated Carbon for Removal of Soluble Pollutants from Water. Filadélfia: ASTM International.

(1994) ASTM D 4607-94: Standard Test Method for Determination of lodine Number of Activated Carbon. Filadélfia: ASTM International. 
(1996) ASTM D 2854-96: Standard Test Method for Apparent Density of Activated Carbon. Filadélfia: ASTM International.

(2002) ASTM D 6851-02: Standard Test Method for Determination of Contact $\mathrm{pH}$ with Activated Carbon. Filadélfia: ASTM International.

(2008) ASTM D 6586-03: Standard Practice for the Prediction of Contaminant Adsorption on GAC in Aqueous Systems Using Rapid Small-Scale Column Tests. Filadélfia: ASTM International.

AMERICAN WATER WORKS ASSOCIATION (AWWA). (2005) Water Treatment Plant Design. 4. ed. Estados Unidos: McGraw-Hill. 972 p.

BANSAL, R. C.; GOYAL, M. (2005) Activated carbon adsorption. Nova: CRC Press. $487 \mathrm{p}$.

BRASIL. (2003a) Agência Nacional de Vigilância Sanitária (ANVISA). Resolução no 165, de 29 de agosto de 2003. Diário Oficial [da] República Federativa do Brasil, Brasília, 2 de setembro de 2003. Disponível em: <http://www.anvisa.gov.br/anvisalegis/resol/2003/ re/165_O3re_3.htm>. Acesso em: 25 fev. 2018.

(2003b) Agência Nacional de Vigilância Sanitária (ANVISA). Resolução no 899, de 29 de maio de 2003. Determina a Publicação do "Guia para validação de métodos analíticos e bioanalíticos". Diário Oficial da União, Brasília.

(2011) Ministério da Saúde. Dispõe sobre os procedimentos de controle e de vigilância da qualidade de água para consumo humano e seu padrão de potabilidade. Portaria no 2.914. Diário Oficial [da] República Federativa do Brasil, Brasília, n. 239, seção 1 , p. 39-46.

CASTILLA, C.M. (2004) Adsorption of organic molecules from aqueous solutions on carbon materials. Carbon, v. 42, n. 1, p. 83-94. https://doi.org/10.1016/j.carbon.2003.09.022

CHINGOMBE, P.; SAHA, B.; WAKEMAN, R.J. (2006) Effect of surface modification of an engineered activated carbon on the sorption of 2,4-dichlorophenoxy acetic acid and benazolin from water. Journal of Colloid and Interface Science, v. 297, n. 2, p. 434-442. https://doi. org/10.1016/j.jcis.2005.10.054

CORWIN, C.J.; SUMMERS, R.S. (2012) Controlling trace organic contaminants with GAC adsorption. Journal of the American Water Works Association, v. 104, n. 1, p. E36-E47. https://doi.org/10.5942/ jawwa.2012.104.0004

CRITTENDEN, J.C.; BERRIGAN, J.K.; HAND, D.W. (1986) Design of rapid small-scale adsorption tests for a constant diffusivity. Journal of Water Pollution Control Federation, v. 58, n. 4, p. 312-319. http:// doi.org/10.2307/25042907

CRITTENDEN, J.C:; BERRIGAN, J.K.; HAND, D.W.; LYKINS, B. (1987) Design of rapid fixed-bed adsorption tests for nonconstant diffusivities. Journal of Environmental Engineering, v. 113, n. 2, p. 243-259.

DEHGHANI, M.; NASSERI, S.; KARAMIMANESH, M. (2O14) Removal of 2,4-Dichlorophenolyxacetic acid (2,4-D) herbicide in the aqueous phase using modified granular activated carbon. Journal of Environmental Health Science and Engineering, v. 12, n. 28, p. 1-10. https://dx.doi.org/10.1186\%2F2052-336X-12-28

DROSTE, R.L. (1997) Theory and practice of water and wastewater. Nova York: John Wiley \& Sons. 816 p.

EBIE, K.; LI, F.; AZUMA, Y.; YUASA, A.; HAGISHITA, T. (2001) Pore distribution effect of activated carbon in adsorbing organic micropollutants from natural water. Water Research, v. 35, n. 1, p. 167-179

ERSAN, G.; KAYA, Y.; APUL, O.G.; KARANFIL, T. (2016) Adsorption of organic contaminants by graphene nanosheets, carbon nanotubes and granular activated carbons under natural organic matter preloading conditions. Science of the Total Environment, v. 565, p. 811-817. https://doi.org/10.1016/j.scitotenv.2016.03.224

FARIA, L.J.S. (2004) Avaliação de diferentes sorventes na extração em fase sólida de pesticidas em água. Desenvolvimento e validação de metodologia. 79f. Dissertação (Mestrado em Química) Universidade Estadual de Campinas, Campinas.

HEIJMAN, S.G.J.; HOPMAN, R. (2009) Activated carbon filtration in drinking water production: model prediction and new concepts. Colloids and Surfaces A. Physicochemical and Engineering Aspects, v. 151, n. 1-2, p. 303-310. https://doi.org/10.1016/S09277757(98)00643-8

HUMBERT, H.; GALLARD, H.; SUTY, H.; CROUÉ, J. (2008) Natural organic matter (NOM) and pesticides removal using a combination of ion exchange resin and powdered activate carbon (PAC) Water Research, v. 42, n. 6-7, p. 1635-1643. https://doi.org/10.1016/j. watres.2007.10.012

INSTITUTO BRASILEIRO DE GEOGRAFIA E ESTATÍSTICA (IBGE). (2015) Indicadores de Desenvolvimento Sustentável, n. 10. Disponível em: <http://biblioteca.ibge.gov.br/visualizacao/livros/ liv94254.pdf>. Acesso em: jul. 2016.

INSTITUTO BRASILEIRO DO MEIO AMBIENTE E DOS RECURSOS NATURAIS RENOVÁVEIS (IBAMA). (2010) Produtos agrotóxicos e afins comercializados em 2009 no Brasil:uma abordagem ambiental. Brasilia: IBAMA. Disponível em: <http://www.ibama.gov.br/phocadownload/ qualidadeambiental/relatorios/produtos_agrotoxicos_comercializados brasil_2009.pdf>. Acesso em: 25 fev. 2018

JARVIE, M.E.; HAND, D.W.; BHUVENDRALINGAM, S.; CRITTENDEN J.C.; HOKASON, D.R. (2005) Simulating the performance of fixedbed granular activated carbon adsorbers: removal of synthetic organic chemicals in the presence of background organic matter. Water Research, v. 39, n. 11, p. 2407-2421. https://doi.org/10.1016/j. watres.2005.04.023

JIANG, H.; ADAMS, C. (2006) Treatability of chloro-s-triazines by conventional drinking water treatment technologies. Water Research, v. 40, n. 8, p. 1657-1667. https://doi.org/10.1016/j. watres.2006.02.013

JIN, X.; PELDSZUZ, S. (2012) Selection of representative emerging micropollutants for drinking water treatment studies: a systematic approach. Science of the Total Environment, v. 414, p.653-663. https://doi.org/10.1016/j.scitotenv.2011.11.035 
MATSUI, Y.; KNAPPE, D.R.U.; IWAKI, K.; OHIRA, H. (2OO2) Pesticide adsorption by granular activated carbon adsorbers. 2. Effects of pesticide and natural organic matter characteristics on pesticide breaktrough curves. Environmental Science and Technology, v. 36, n. 15, p. 3432-3438. https://doi.org/10.1021/ esO11366u

MATSUI, Y.; KNAPPE, D.R.U.; TAKAGI, R. (2002) Pesticide adsorption by granular activated carbon adsorbers. 1. Effects of natural organic matter preloading on removal rates and model simplification. Environmental Science and Technology, v. 36, n. 15, p. 3426-3431. https://doi.org/10.1021/es0113652

MWH. (2005) Water Treatment: principles and design. 2. ed. Estados Unidos: John Wiley \& Sons. 1968 p.

PESTICIDE ACTION NETWORK (PAN). (2010) PAN Pesticide Database. San Francisco: Pesticide Action Network. Disponível em: <http:www.pesticideinfo.org>. Acesso em: 21 mar. 2017.

REN, L.; ZHANG, J.; LI, Y.; ZHANG, C. (2O11) Preparation and evaluation of cattail fiber-based activated carbon for 2,4-diclorophenol and 2,4,6-triclorophenol removal. Chemical Engineering Journal, v. 168, n. 2, p. 553-561. http://dx.doi.org/10.1016/j.cej.2011.01.021

SALMAN, J.M.; NJOKU, V.O.; HAMEED, B.H. (2011) Batch and fixedbed adsorption of 2,4-dichlorophenoxyacetic acid onto oil palm frond activated carbon. Chemical Engineering Journal, v. 174, n. 1, p. 33-40. https://doi.org/10.1016/j.cej.2011.08.024

SCHARF, R.G.; JOHNSTON, R.W.; SEMMENS, M.J.; HOZALSKI, R.M. (2010) Comparison of batch sorption tests, pilot studies, and modeling for estimating GAC bed life. Water Research, v. 44, n. 3, p. 769-780. https://doi.org/10.1016/j.watres.2009.10.018

SCHIDEMAN, L.C.; SNOEYINK, V.L.; MARIÑAS, B.J.; DING, L.; CAMPOS, C. (2007) Application of a three-component competitive adsorption model to evaluate and optimize granular activated carbon systems. Water Research, v. 41, n. 15, p. 3289-3298. https:// doi.org/10.1016/j.watres.2007.05.007

SILVERSTEIN, R.M.; WEBSTER, F.X.; KIEMLE, D.J. (2010) Identificação espectrométrica de compostos orgânicos. 7. Ed. Rio de Janeiro: LTC.

SNOEYINK, V.L.; SUMMERS, R.S. (1999) Adsorption of Organic Compounds by activated Carbon. In: LETTERMAN, R.D. Water Quality and treatment: a handbook of community water supplies. 5. ed. Estados Unidos: McGraw-Hill. 1248 p.

STACKELBERG, P.E.; GIBS, J.; FURLONG, E.T.; MEYER, M.T.; ZAUGG S.D.; LIPPINCOTT, R.L. (2007) Efficiency of conventional drinkingwater-treatment processes in removal of pharmaceuticals and other organic compounds. Science of the Total Environment, v. 377, n. 2-3, p. 255-272. https://doi.org/10.1016/j.scitotenv.2007.01.095 